\title{
Multichannel Quantum Defect Theory Analysis of Overlapping Resonance Structures in Lu-Fano Plots of Rare Gas Spectra
}

\author{
Chun-Woo Lee and Ja-Hyun Kong

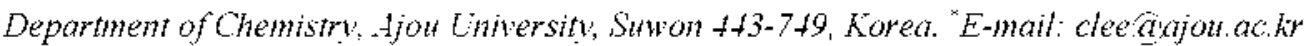 \\ Received Jine 08, 2009, Accepted Jine 28. 2009
}

\begin{abstract}
Although overlapping resonances have been studied extensively in conventional resonance theories, there have not been many studies on them in multichannel quantum defect theories (MQDT). In MQDT, overlapping resonances occur between the channels instead of states, which pose far greater difficulty. Their systematic treatment was obtained for cases involving degenerate closed channels by apply ing our previous theory, which decouples backoround scattering from the resonance scattering in the MQDT formulation. The use of mathematical theory on con-diagonalization and con-sinilarity was essential for handling the non-Hermitian symmetric complex matrix. Overlapping resonances in rare gas spectra of $\mathrm{Ar}, \mathrm{Kr}$ and Xe were analyzed using this theory and the results were compared with the ones of the previous altemative parameterizations of MQDT which make the open-open part $K^{* i 0}$ and closed-closed part $K^{-i c}$ of reactance submatrices zero. The comparison revealed that separation of background and resonance scatterings achieved in our formulation in a sy stematic way was not achieved in the representation of $K^{\circ \circ}=0$ and $K^{\circ c}=0$ when overlapping resonances are present.
\end{abstract}

Key Wonds: MQDT, Overlapping resonance, Phase renormalization, Lu-Fano plot, Rare gas spectra

\section{Introduction}

Although multichannel quantum defect theory (MQDT) is a powerful theory of resonance that can describe complex spectra including both bound and continuum regions with only a few parameters, ${ }^{1 \cdot 3}$ the resonance structures were not identified transparently in its formulation due to the indirect treatment of resonance. A special treatment is needed to identify the resonance terms. Such treatments include the phase renormalization considered long ago by Eissner et $a l^{4}$ introduced by Cooke and Cromer to obtain more effective representation and independently by Giusti-Suzor and Fano." Since resonance shows up only when closed channels are present and are coupled to open channels. the main focus was on obtaining a pure collpling term between the open and closed channels.

In different directions, efforts to reformulate MQDT into forms with a one-to-one correspondence to those in Fano's configuration mixing theory of resonance ${ }^{1]}$ revealed that more elaborate process of disentangling background and resonance scatterings is needed to analyze resonance structures in the MQDT formulation. ${ }^{13-15}$ This work is part of an ongoing investigation that extends the previous theory to the cases involving degenerate closed channels. These latter cases are interesting in that it is the simplest system where overlapping resonances are present and need to be handled properly. Not many MQDT studies ${ }^{*}$ of overlapping resonances are present in contrast to the huge amounts of work in a variety of disciplines in the conventional theory of resonance. ${ }^{16-34}$ In the formulations of MQDT. perturbations between Ryddberg series. identification of an interloper in complex resonances, vanishing widths and stabilization of some excited levels. relations between the number of $q$ reversals and perturbers were the phenomena studied related to a overlapping resonance.

This study refined the MQDT analy'sis of overlapping resonances by disentangling the background scatterings from the resonance ones using the technique developed by this group. ${ }^{15}$ This theory was applied to the photo-absorption and ionization spectra of rare gases. where ample theoretical MQDT studies and experimental work have been carried out. ${ }^{25.26}$ Ueda, Lecomte. Giusti-Suzor and Fano and Mullins also adopted the same alternant parameterization scheme to treat degenerate closed channels. ${ }^{9,27.39}$ In particular. Ueda succeeded in find the phase renormalization and orthogonal transformations that make the open-open part $K^{\circ \infty}$ and closed-closed part $K^{-\infty}$ of reactance sub-matrices zero for the systems involving degenerate closed channels. However, we will show in this work that making $K^{-i \omega}$ and $K^{-c c}$ null does not guarantee the separation of background and resonance scatterings and identification of resonance structures in the presence of overlapping resonances. Also. our formulation provides the phy sical background to his work of using conplex quantum defects and removes the ambiguities in his approaches.

\section{Summay of the Previous Results}

Before describing the alternant parameterization scheme to treat degenerate closed channels. let us first summarize our previous formulation. ${ }^{15}$ Let the scattering matrix

$$
S=\left(\begin{array}{ll}
S^{\mathrm{oo}} & S^{\mathrm{oc}} \\
S^{\mathrm{co}} & S^{\mathrm{cc}}
\end{array}\right)
$$

describe the photo-fragmentation (including dissociation and ionization) processes in the intermediate range along the fragmentation coordinate. The super-indices, $o$ and $c$. used for the S"s sub-matrices denote the open and closed channels, respectively. Note that the classification of channels as open or closed is meaningful only at a large $R$. Nevertheless. it may still be convenient to keep this classification in the interme- 
diate range. Although all the charnels are needed to describe the motion in the intermediate range. some have become closed and no longer exist in the limit of $R \rightarrow \infty$. Therefore. open channels are only needed to describe the transition probability amplitudes in various photo-fragmentation processes. The physical scattering matrix, $\boldsymbol{S}$, which gives the probability amplitudes. is obtained as follows:

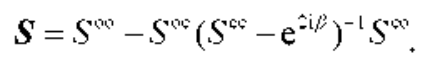

where $\beta$ is a quantum defect parameter used for the base pair of closed channels as $\mp\left(m_{j} / \pi \kappa_{j}\right)^{1:} \exp \left( \pm \mathrm{i} \beta_{3}\right)\left(D_{j} f_{l}^{+} \pm \mathrm{i} D_{3}^{-1} f_{3}^{-}\right) / 2$. where $f_{i}=$ denotes $\exp \left( \pm \mathrm{i} k_{j} R\right)$. It is given by $\pi(-l+1)$ for the Coulomb fields. ${ }^{2}$ The remaining definitions of the parameters in the closed channel base pairs can be found in Ref. [2]

For the background scattering process, an 'effective' scattering sub-matrix $\sigma^{\infty}$ was introduced to supersede $S^{\infty}$. which is defined in terms of the reactance sub-matrix, $K^{-\infty}$. as $\left(1-i K^{\infty}\right)$ $\left(1+i K^{(x)}\right)^{-1}$ and simultaneously diagonalizable with $K^{-\infty \infty}{ }^{14}$ (Note that $S^{+\infty}$ is taken as a complex conjugate of the usual definition for convenience in studying autoionization.) In terms of this effective scattering matrix the physsical scattering matrix can be expressed as follows:

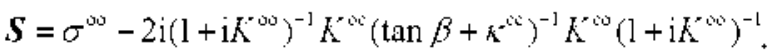

where $\kappa^{\text {coc }}$ is defined as $-\mathrm{i}\left(1-S^{\mathrm{cc}}\right)\left(1+S^{\circ c}\right)^{-1}$ and is used extensively by Lecomte. ${ }^{9}$ Since $\sigma^{\infty}$ and $K^{+\infty}$ can be diagonalizable simultaneously. they can be expressed as $\sigma^{\infty \times}=U e^{-2 i \sigma^{-6}} V^{\mathrm{T}}$ and $K^{-\infty}=U \tan \delta^{O} U^{\mathrm{T}}$. By substituting these into $\mathrm{Eq}$. (3). the following is obtained

$$
\begin{aligned}
\boldsymbol{S} & =U \mathrm{e}^{-\mathrm{i} \hat{\varepsilon}^{30}}\left[1+2 \mathrm{i} \xi\left(\tan \beta+\kappa^{-0 \mathrm{C}}\right)^{-1} \xi^{\mathrm{T}}\right] \mathrm{e}^{-\mathrm{i} \delta^{0}} U^{\mathrm{T}} \\
& \equiv U \mathrm{e}^{-1 \delta^{0}} S_{\mathrm{r}} \mathrm{e}^{-1 \delta^{0}} U^{\mathrm{T}} .
\end{aligned}
$$

where $=$ denotes $\cos \delta^{\circ} U^{\mathrm{T}} \mathrm{K}^{\circ \infty}$ and the part inside the bracket is denoted as $\boldsymbol{S}_{\mathrm{r}}$.

Consider extracting the resonance structures contained in Eq. (4). The resonance stnictures in the physical scattering matrix can best be seen in the behavior of its eigenphase shifts $\delta_{j}$ because they undergo rapid changes in the neighborhood of a resonance. The avoided interactions between the different eigenphase shifts $\delta_{j}$ also affect their behaviors. obscuring the resonance stnictures. but can be removed easily by considering the eigenphase sum. The eigenphase sum $\delta_{\Sigma}\left(=\Sigma, \delta_{y}\right)$ can be obtained by calculating the determinant of the plysical scattering matrix: $\operatorname{det}(S)=\exp \left(-2 i \delta_{-}\right)$. From

$$
\begin{aligned}
\operatorname{det}(S) & =\operatorname{det}\left(\sigma^{\infty}\right) \frac{\operatorname{det}\left(\tan \beta+\kappa^{\infty} *\right)}{\operatorname{det}\left(\tan \beta+\kappa^{\infty}\right)} \\
& =\operatorname{det}\left(\sigma^{\infty}\right) \frac{\tan \beta+\tan \Delta^{\circ *}}{\tan \beta+\tan \Delta^{\circ}},
\end{aligned}
$$

$\delta_{\Sigma}=\delta_{\Sigma}+\delta_{1}$ is obtained. The last equality in Eq. (5) is valid only for the cases involving 1 closed channel. $J^{\circ}$ is the complex phase shift considered by Dubau and Seaton ${ }^{\text {sic }}$ and is defined as $\delta^{\infty}-\mathrm{i}_{j}^{c}$. The unitary factorization performed in $\mathrm{Eq}$. (5) still falls short of decoupling the background scattering from the resonance one. In order to remove the background contribution from $\delta_{\mathrm{r}}$ completely phase renormalization $\hat{\delta}_{\mathrm{r}}=\delta_{\mathrm{r}}$ $-\pi t_{\mathrm{T}}$ in the resonance eigenchannel needs to be performed for $\delta_{\mathrm{t}}$. The extent of renonnalization is determined by the coupling strength ${ }^{2}$ "between the open and closed channels in the "tilde" representation which is devoid of elastic potential scattering and is given by $\tan \pi \mu_{1}=-\xi^{2}$ tan $\delta^{\infty}$. For more detailed information. please refer to Ref [15]. In the tilde representation, where the complete disentanglement of background scattering from resonance scattering is attained. the relation

$$
\tan \left(\delta_{\Sigma}-\delta_{\Sigma}^{0}\right)\left[\tan \beta+\Re\left(\kappa^{\infty}\right)\right]=\Im\left(\kappa^{\infty i}\right)=-\xi^{2}
$$

can be simplified to

$$
\tan \tilde{\delta}_{\Sigma} \tan \bar{\beta}=\Im\left(\tilde{\kappa}^{c s}\right)=-\bar{\xi}^{2} .
$$

and the coupling parameters $\xi$ and $\tilde{\xi}$ between the open and closed channels in respective original and tilde representations are related by the simple equation.

The parameters described thus far were extracted from the

\begin{tabular}{|c|c|c|}
\hline$\delta^{c}$ & $\eta^{\mathrm{c}}$ & $\delta_{\varepsilon}^{\mathrm{B}}$ \\
\hline 0.95 & 0.0073 & 0.93 \\
\hline $\begin{array}{l}. i^{\circ} \\
0.30\end{array}$ & $\begin{array}{c}\mu_{\mathrm{r}} \\
-0.0032\end{array}$ & $\begin{array}{l}\mu_{s}^{\prime \prime} \\
0.29\end{array}$ \\
\hline $\begin{array}{l}\Re\left(a^{20}\right) \\
1.40\end{array}$ & $\begin{array}{l}\tilde{s}\left(k^{*}\right) \\
-0.021\end{array}$ & $\begin{array}{c}\underbrace{2} \\
0.021\end{array}$ \\
\hline$\frac{\Re\left(\tilde{K}^{\infty}\right)}{0}$ & $\begin{array}{l}3\left(\kappa^{\infty}\right) \\
-0.0073\end{array}$ & $\begin{array}{c}\xi^{2} \\
0.0073\end{array}$ \\
\hline
\end{tabular}
Lu-Fano plots ${ }^{2}$ for the excited levels of two Rydberg series of

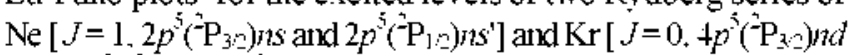
and $4 p^{5}\left({ }^{2} \mathrm{P}_{1}\left(\mathrm{C}_{2}\right) n s^{6}\right]^{6}$ obtained from the NIST Atomic Spectra Database ${ }^{+1}$ and are summarized in Tables $I$ and 2 and shown

\begin{tabular}{|c|c|c|}
\hline $\begin{array}{c}\delta^{\circ} \\
1.301\end{array}$ & $\begin{array}{c}\dddot{i}^{*} \\
0.0185\end{array}$ & $\begin{array}{l}\delta_{\Sigma}^{\mathrm{B}} \\
1.53\end{array}$ \\
\hline $\begin{array}{c}t^{*} \\
0.41\end{array}$ & $\begin{array}{c}\mu_{\mathrm{r}} \\
-0.0212\end{array}$ & $\begin{array}{c}\mu_{\Sigma}^{\circ} \\
0.4659\end{array}$ \\
\hline $\begin{array}{l}\mathfrak{H}\left(k^{* c}\right) \\
3.60\end{array}$ & $\begin{array}{l}\tilde{s}\left(K^{\mathrm{cs}}\right) \\
-0.26\end{array}$ & $\begin{array}{c}\xi^{2} \\
0.26\end{array}$ \\
\hline $\begin{array}{c}\mathfrak{M}\left(\hat{\kappa}^{\mathrm{CS}}\right) \\
0\end{array}$ & $\begin{array}{l}\tilde{s}\left(\tilde{K}^{w}\right) \\
-0.018\end{array}$ & $\begin{array}{c}\tilde{\xi^{2}} \\
0.018\end{array}$ \\
\hline
\end{tabular}

Table 1. Dynamic parameters extracted from the Lu-Fano plot for the neon Rydberg series with $J=1\left(2 p^{5}{ }^{3} \mathrm{P}_{32} n s\right.$ and $\left.2 p^{5}{ }^{2} \mathrm{P}_{1: 2} n s^{\prime}\right)$

Table 2. Dynamic parameters extracted from the L Ll-Fano plot the two interaction Rydberg series of $\mathrm{KI}$ with $J=0\left(4 p^{5}{ }^{2} \mathrm{P}_{3: 1}\right) n d$ and $4 p^{5}$ $\left.{ }^{2} \mathrm{P}_{1: 2} n s^{\prime}\right)$ 
Lu-Fano plot of $\mathrm{Ne}$

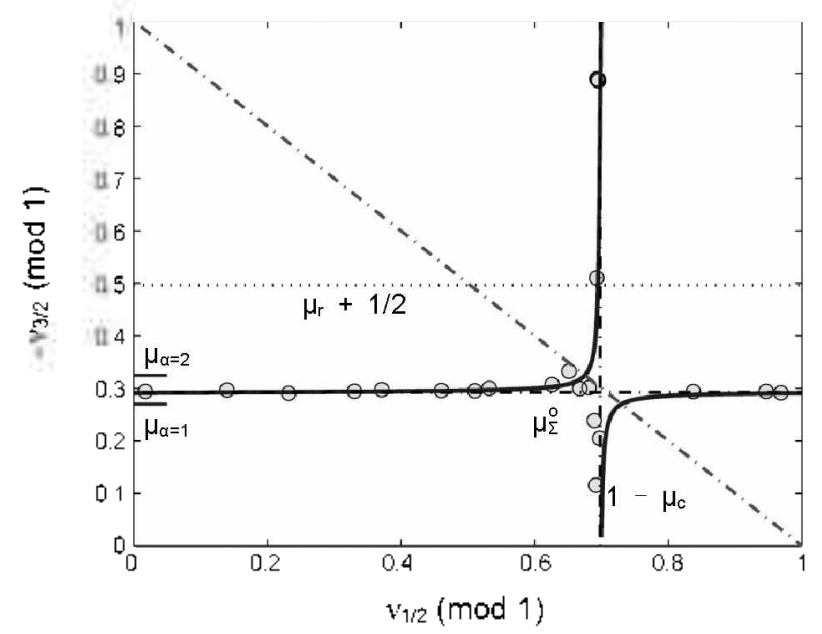

Figure 1. Lu-Fano plot for the neon Rydberg series with $J=1\left(2 p^{5}\right.$

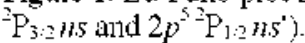

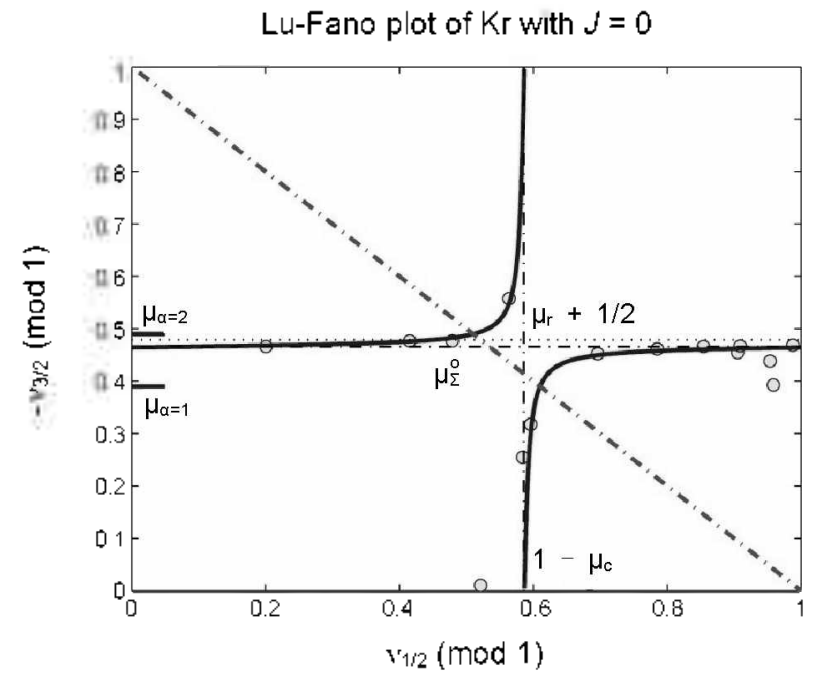

Figure 2. Lu-Fano plot for the two interaction Rydberg series of Kr with $J=0\left(4 p^{52} \mathrm{P}_{3: 2} n d\right.$ and $\left.4 p^{5}{ }^{3} \mathrm{P}_{1: 2} n s^{\prime}\right)$.

graphically in Figs. I and 2. Note that in both cases. the values of $z^{-2}$ are much smaller than those of $\Sigma^{2}$. which means caution should be taken not to use $\xi^{2}$ to represent the coupling strength between the open and closed channels. A cursory look at the Lu-Fano plot immediately shows weak coupling between the two channels and confirms the correctness of the small value of

Overdapping Resonances for the System Involving Degenerate Closed Channels. Consider the case where closed channels are degenerate. as in the photo-absorption spectra of the noble gases. Ar to Xe. which were used as a testing ground for analyzing the multichannel phenomena. ${ }^{2.5636}$ Since noble gases have $a p^{61} \mathrm{~S}$ ground state. photo-absorption excites their atoms to $J=1$. which are odd parity channels with a $p^{5} \mathrm{P}^{\circ}$ core. The ionization channels have $l_{l}=0$ or 2 and $j=1 / 2.3 / 2$. $5 / 2$. The three values of $j_{i}$ combined with the $J_{i}=1 / 2$ and $3 / 2$ for the core yield 5 channels with $J=1$, where 3 channels have $E_{j}=E_{32}$ and 2 have $E_{j}=E_{1:}$.

Before describing the general approach. let us start from the

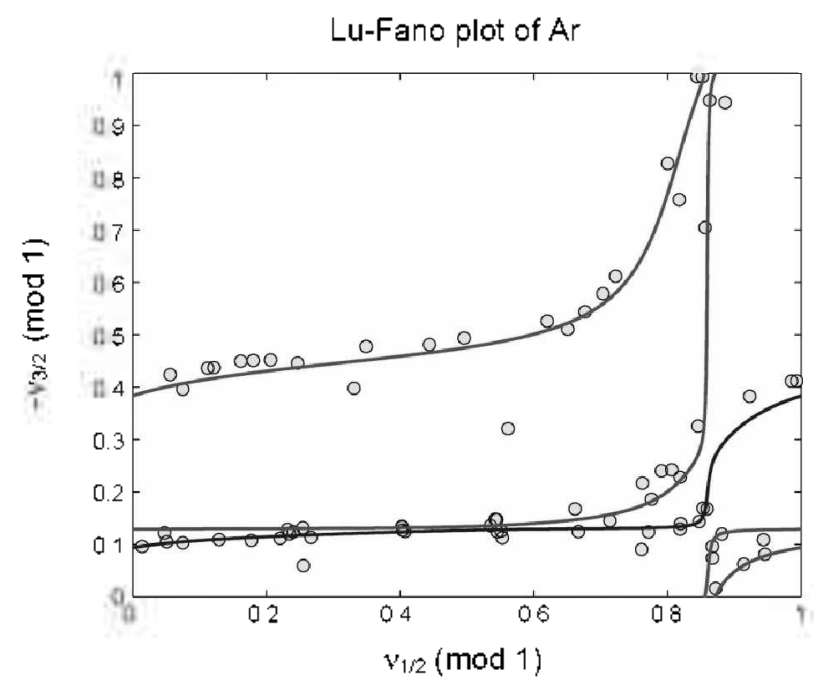

Figure 3. Lul-Fano plot for the $J=1$ states of argon at the lowest ionization thresholds ${ }^{2} \mathrm{P}_{3: 2}^{0}$ and ${ }^{2} \mathrm{P}_{1: 2}^{0}$.

straightforward formulation where the background and resonance scatterings remain intertwined but separate the two terms contributing to a resonance so that the resonance structures can be identified. It starts from obtaining the phase $\delta_{\mathrm{r}}$ of $\operatorname{det}\left(\tan \beta+\kappa^{* i x}\right)$ by calculating $\tan \delta_{\mathrm{t}}$ from the ratio of its real and imaginary parts. The real part can be obtained as a quadratic polynonual of $\tan \beta$ and the intaginary one as a linear polynomial of $\tan \beta$. If $r_{1}$ and $\mu_{2}$ are two roots of the real part. $\tan \delta_{\mathrm{r}}$ can be expressed as follows:

$$
\tan \delta_{\mathrm{r}}=\frac{s_{1}}{\tan \beta+y_{1}}+\frac{s_{2}}{\tan \beta+r_{2}} .
$$

Two roots are obtained as $1 / 2\left\{\operatorname{tr} \Re\left(\kappa^{c c}\right) \pm \sqrt{D}\right\}$, where $D$ denotes $\left[\operatorname{tr} \Re\left(k^{c c}\right)\right]^{2}-4 \operatorname{det} \Re\left(k^{c c}\right)+4$ det $\Im\left(\kappa^{c c}\right)$. Sinilarly. the expressions for $s_{1}$ and $s_{2}$ can be obtained in a straightforward manner and are obtained as follows:

$$
\begin{aligned}
& \left(\begin{array}{l}
s_{1} \\
s_{2}
\end{array}\right)=\frac{1}{2 \sqrt{D}}\left(\begin{array}{c}
\Delta \Re\left(\kappa^{\infty}\right) \Delta \Im\left(\kappa^{\infty}\right)+\sqrt{D} \operatorname{tr} \Im\left(\kappa^{\infty}\right) \\
-\Delta \Re\left(\kappa^{c c}\right) \Delta \Im\left(\kappa^{c c}\right)+\sqrt{D} \operatorname{tr} \Im\left(\kappa^{c c}\right)
\end{array}\right. \\
& +4 \Re\left(\kappa_{12}^{c c}\right) \Im\left(\kappa_{12}^{c c}\right) \\
& \left.-4 \Re\left(\kappa_{12}^{c c}\right) \Im\left(\kappa_{12}^{c c}\right)\right) \text {. }
\end{aligned}
$$

where $\Delta \Re\left(\kappa^{* c}\right)$ and $\Delta \widetilde{s}\left(\kappa^{c c}\right)$ denote the real and inaginary parts of the difference. $k_{11}^{c c}-\kappa_{22}^{c c}$ of the diagonal elements of $\kappa^{\text {ic }}$. respectively. If a single closed chanmel is involved. only one tenn will appear on the right hand side of Eq. (8). Therefore. each term on the right-hand side of $\mathrm{Eq}$. (8) might be interpreted as $\tan \delta_{\mathrm{rl}}$ and $\tan \delta_{\mathrm{r} \text { ? }}$ as done in Ref. [11]. Fig. 4 shows $\delta_{\mathrm{rl}} . \delta_{\mathrm{T}_{2}}$ and $\delta_{\mathrm{T}}$ obtained in this manner from the Lu-Fano plot of Ar in Fig. 3. A detailed discussion of the graphs will be given later but there was a problem with the interpretation described thus far in that not only is $\delta_{\mathrm{t}}$ different from $\delta_{1]}+\delta_{\mathrm{t}}$ but there is also no way that $\tan \delta_{\mathrm{r}}$ can be equal to $\tan \delta_{\mathrm{r}]}+\tan \delta_{\mathrm{r} 2}$. 
Eigenquantum-defect sum of $\mathrm{Ar}$

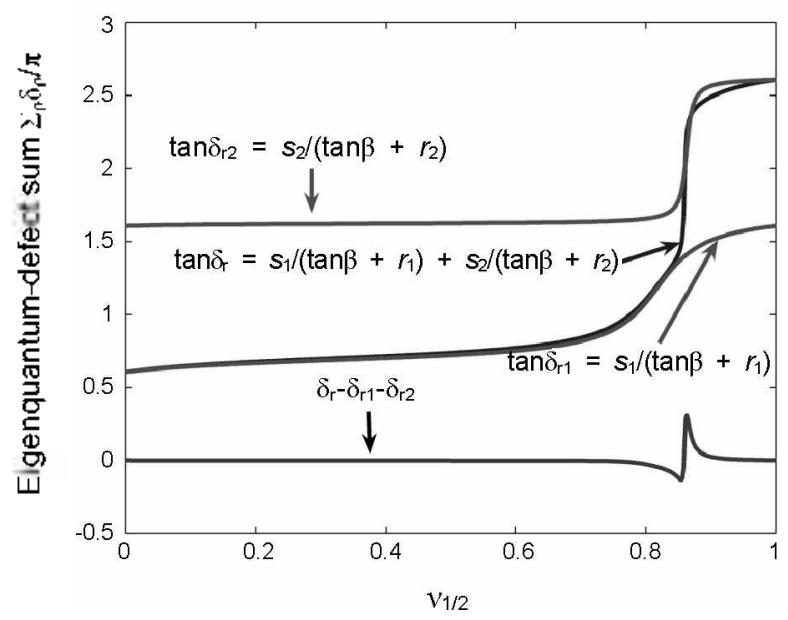

Figure 4. Decomposition of the sum of the eigenquantum-detects extracted from the Lu-Fano plot for the $J=1$ states of argon at the lowest ionization thresholds ${ }^{2} \mathrm{P}^{n}$. and ${ }^{2} \mathrm{P}_{\mathrm{l}}$.

This problem can be avoided if symmetric $k^{-"}$ can be diagonalized by a similarity transformation, $V_{K}{ }^{50} F^{r}$, with a real orthogonal matrix $r$. Unfortunately, this cannot be achieved in general because $\kappa^{\infty}$ is not a normal matrix $x^{\text {t2 }}$ but a complex symmetrical one. Nevertheless. it can be diagonalized as $I K^{\text {cc }} F^{-T}$ with a complex unitary matrix $I$. Such a diagonalization is known as Takagi's factorization or more formally condiago-

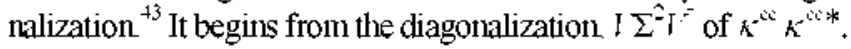
where $\Sigma$ is a diagonal matrix, whose elements $\sigma_{i}(j=1.2, \ldots)$

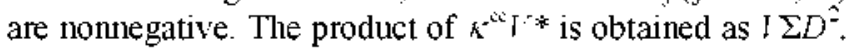
where $D$ is a diagonal matrix whose $j$-th element is given by $\exp \left(\mathrm{i} \theta_{3}\right)$ with $\theta_{3}$ real. $\sigma_{t} D_{3}^{\hat{\gamma}}$ is called a coneigenvalue with the coneigenvector $v_{\text {, which }}$, comprises $\mathrm{I}^{\prime}$ as $\left[v_{1} \cdots v_{\mathrm{B}}\right]$. Unlike eigenvalues, they are not defined uniquely because $\mathrm{e}^{1 a_{j}} \sigma_{j} D_{j}^{2}$ is also a coneigensalue for all real $\alpha_{t}$

Using this theory of consimilarity and condiagonalization. $K^{\text {cc }}$ is Takagi-factorized into $I \Sigma D^{-2} T^{-T}$. It can be given into a factorized form as $U \Sigma U^{\mathrm{T}}$ with $U=I D$. Using the complex phase shifts $J_{j}^{c}$ defined as $\delta_{j}^{c}-\mathrm{ir}_{j i}^{c}$ the diagonal matrix $\Sigma D^{2}$ may be denoted as $\tan J^{\circ}$ whereby $\kappa^{\text {ic }}$ can be expressed as follows:

$$
\kappa^{c c}=I^{\top} \tan \Delta^{c} F^{-T} \text {. }
$$

Note that $\tan \beta+\tan \Delta^{c}$ cannot be obtained from $\tan \beta+\kappa^{* \text { ic }}$ through a unitary transformation and is not a conjugate of it because $I^{-T}$ I differs from unity. A conjugation relation is obtained when $\mathrm{F}$ ' is a real orthogonal matrix, which in turn requires $\kappa^{\text {"in }}$ to be a normal matrix. If this holds. $\tan \beta+\kappa^{\text {"in }}$ would be a conjugate to $\tan \beta+\tan \Delta^{c}$ as $\tan \beta$ is a constant matrix for the case involving degenerate close channels and equal to $I\left(\tan \beta+\tan \Delta^{c}\right) V^{-1}$ whereby $\operatorname{det}\left(\tan \beta+\kappa^{\text {cic }}\right)$ would become $\Pi_{j=1}\left(\tan \beta+\tan \Delta_{i j}^{c}\right)$. In this case. the phase $\delta_{1}^{\text {lu। }}$ of $\operatorname{det}(\tan \beta+$ $\kappa^{(i)}$ ) could be obtained as a simple sum of tenns $\delta_{I j}^{\text {id }}$ from each eigenchannel satisfying

$$
\tan \delta_{\mathrm{rj}}^{\mathrm{icl})}=\Im\left(\tan \Delta_{j}^{\mathrm{c}}\right) /\left[\tan \beta+\Re\left(\tan \Delta_{j}^{\mathrm{c}}\right)\right]
$$

without any coupling terns, which means that the resonances in each eigenchannel are isolated.

However. $\kappa^{-i 2}$ is generally not a normal matrix and $\delta_{\Gamma}^{(d)}$ is no longer obtained as a sum of $\delta_{1 ;}^{\text {,d }}$ but contains a coupling term. The resonances in different channels are no longer independent of each other and are overlapped. Since $r^{-\mathrm{T}} \mathrm{r}^{\prime}$ is unity when $k^{\text {iv }}$ is a normal matrix so that the resonances are isolated. the overlapping resonance can be dealt by a deviation of ${ }^{\prime} \cdot{ }^{\top} \mathrm{T} \cdot$ from unity. If the deviation $I^{\mathrm{T}} I-I$ is denoted as $\Delta, \operatorname{det}(\tan \beta+$ $K^{* i}$ ) can be expanded in terms of it using Cayley's theorem ${ }^{44}$ for the system of degenerate closed channels as follows:

$$
\begin{aligned}
& \operatorname{det}\left(\tan \beta+\Gamma^{\prime} \tan \Delta^{\mathrm{c}} \Gamma^{\mathrm{T}}\right)=\operatorname{det}\left(\tan \beta+\Gamma^{\mathrm{T}} \Gamma^{\prime} \tan \Delta^{\mathrm{c}}\right) \\
& \quad=\prod_{l}\left(\tan \beta+\tan \Delta_{j}^{\mathrm{c}}\right) \\
& \quad+\sum\left(\tan \beta+\tan \Delta_{?}^{\mathrm{c}}\right) \operatorname{cof}_{2,}\left(\Delta I \tan \Delta^{\mathrm{c}}\right)+\cdots .
\end{aligned}
$$

where the matrix identity $\operatorname{det}(I-U T)=\operatorname{det}(I-I U)$ and the constancy of $\tan \beta$ for the degenerate closed channels were used for the first equality. Note that $\tan \beta+J^{T} T^{\top} \tan \Delta^{c}$ is different from $I^{T}\left(\tan \beta+{ }^{* * 1}\right) r$. If closed channels were not degenerate. the above procedure could not be applied. In that case, diagonalization of $\tan \beta+\kappa^{* N}$ instead of $\kappa^{* N}$ could be done to systematically obtain an overlapping resonance term and terms beyond it. The latter case will not be treated in this paper. The first term on the right-hand side of Eq. (12) is the form of a product of terms corresponding to isolated resonances described by Eq. (11). The remaining terms on the right-hand side of Eq. (12) describe the effect beyond the isolated resonances and correspond to overlapping ones. Therefore, Takagi's factorization provides a means of describing an overlapping resonance in the context of MQDT.

For this use of the factorization the uniqueness problem of the factorization should be cleared up first. For example, factorization can be performed in either of two ways. $U \Sigma U^{\mathrm{T}}$ or $\Gamma \Sigma D^{2} I^{\mathrm{T}}$. Consider $U^{\mathrm{T}} U$ and $I^{\cdot \mathrm{T}} F^{:}$. Note that $\left|\operatorname{det}\left(I^{-{ }^{T}} I\right)\right|=\left|\operatorname{det}\left(C^{\mathrm{T}} U\right)\right|=1$ as both $U$ and $I$ are unitary. But. their traces are different from the number of closed channels. $\operatorname{tr}\left(I^{c c}\right)$. To make the problem easy' let us consider a sy'stem of two closed channels for which $\operatorname{tr}\left(I^{c c}\right)=2$. In this case. any unitary matrix can be expressed as $\exp [i(a+b \boldsymbol{\sigma} \cdot \mathbf{c})]$ with Pauli matrices $\boldsymbol{\sigma}$. If we take $r^{\prime}$ as exp $(\mathrm{i} b \boldsymbol{\sigma} \cdot \mathbf{c})$ with $\exp (\mathrm{i} a)$ taken away with a suitable choice of $D$. $r^{\text {T }} F^{\prime}$ takes especially a simple form, that is, its off-diagonal elements are purely imaginary and its two diagonal elements are complex conjugate with each other so that its trace is real. ${ }^{+5.16}$ The latter property makes the $\operatorname{trace} \operatorname{tr}\left(I^{\mathrm{T}} \mathrm{V}\right)\left[=\operatorname{tr}\left(I^{\prime} T^{\mathrm{T}}\right)\right]$ a nice candidate for a parameter showing the extent of overlapping of resonances. We will use this quantity in the next section as a barometer to show the extent of overlapping of resonances. Note that matrix relations are not invariant under con-similarity transformation. For example. the matrix relation $\kappa^{* N}=-\mathrm{i}(\mathrm{l}-$ $\left.S^{\infty c}\right)\left(1+S^{c c}\right)^{-1}$ does not hold between the con-diagonalized $k^{\text {ic }}$ and $S^{\infty}$. Although it may be considered peculiar at first. actually it might be more physically reasonable. We want to investigate this point further in the future.

Let us consider the decoupling of background and resonance terms in the con-diagonalized tan $\Delta^{\circ}$ of $k^{\circ}$. Using the general 
Table 4. Extraction of the dynamic parameters for the $J=1$ states of

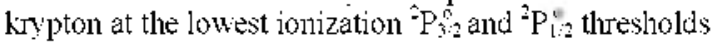

\begin{tabular}{|c|c|c|c|c|c|}
\hline \multicolumn{2}{|c|}{$\begin{array}{l}\text { Original } \\
\text { representation }\end{array}$} & \multicolumn{2}{|c|}{$\begin{array}{l}\text { Con-diagonalized } \\
\text { representation }\end{array}$} & \multicolumn{2}{|c|}{$\begin{array}{c}K^{m}=0 \text { and } K^{\varphi \varphi}=0 \\
\text { representation }\end{array}$} \\
\hline$r_{1}$ & $r_{2}$ & $\mathfrak{R}\left(k_{[1}^{\text {cecidi })}\right)$ & $\mathfrak{R}\left(\kappa_{2:}^{i(j)}\right)$ & $r_{l}$ & $r_{2}$ \\
\hline 0.71 & 0.34 & 0.71 & 0.34 & 0.73 & 0.34 \\
\hline$s_{1}$ & $s_{2}$ & 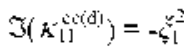 & $\Im\left(\kappa_{11}^{(i i)}\right)=-\xi_{2}^{2}$ & $s_{1}$ & $s_{2}$ \\
\hline \multirow[t]{2}{*}{-0.20} & -0.020 & -0.21 & -0.015 & -0.21 & -0.018 \\
\hline & & & & 0.10 & $\begin{array}{l}d_{2} \\
1.00\end{array}$ \\
\hline \multicolumn{6}{|c|}{ 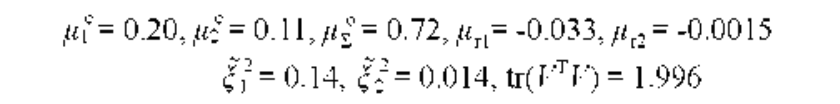 } \\
\hline
\end{tabular}

Table 5. Extraction of the dynanic parameters for the $J=1$ states of xenon at the lowest ionization ${ }^{2} \mathrm{P}_{3: 2}^{0}$ and ${ }^{2} \mathrm{P}_{1: 2}^{\mathrm{N}}$ thresholds

\begin{tabular}{|c|c|c|c|c|c|}
\hline \multicolumn{2}{|c|}{$\begin{array}{c}\text { Original } \\
\text { representation }\end{array}$} & \multicolumn{2}{|c|}{$\begin{array}{l}\text { Con-diagonalized } \\
\text { representation }\end{array}$} & \multicolumn{2}{|c|}{$\begin{array}{c}K^{\infty}=0 \text { and } K^{\circ 9}=0 \\
\text { representation }\end{array}$} \\
\hline$\mu_{l}$ & $\mathrm{~F}_{2}$ & $\mathfrak{R}\left(k_{[1}^{-c e(d)}\right)$ & $\mathfrak{R}\left(k_{2=}^{c y}(d)\right)$ & $r_{l}$ & $r_{2}$ \\
\hline 1.00 & 0.074 & 0.99 & 0.077 & 1.14 & 0.075 \\
\hline$s_{l}$ & $s_{2}$ & 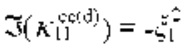 & $\widetilde{s}\left(\kappa_{11}^{\cos (d)}\right)=-\sigma_{2}^{2}$ & $s_{l}$ & $s_{2}$ \\
\hline-0.52 & -0.0069 & -0.52 & -0.0052 & -0.57 & -0.006 \\
\hline & & & & $\begin{array}{c}n_{2} \\
0.28\end{array}$ & $\begin{array}{l}d_{2} \\
1.00\end{array}$ \\
\hline \multicolumn{6}{|c|}{ 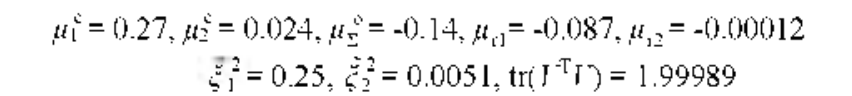 } \\
\hline
\end{tabular}

there. By this rearrangement of dynamics by phase renomalization $\mu^{\circ}$ and orthogonal transformation $W^{\circ c}$, the constant $\tan \beta$ becomes non-diagonal $H^{\circ c} \tan \left(\beta+\pi t^{c}\right) W^{c c \mathrm{~T}}$ and the isolation of overlapped resonances is not attained

Since the difficulty in disentangling background from resonance couplings in the null $K^{-\infty}$ and $K^{-c c}$ representation arose from the impossibility of the disentanglement of background and resonance scatterings when there is overlapping resonances. it was avoided in the present work by limiting the application of making $K^{+\infty}$ and $K^{\text {Ev }}$ null to the normal matrix part that is devoid of overlapping resonances. Such a restrictive application requires decomposition of $\tan \beta+K^{-e c}$ into the normal and nonnormal matrix parts. which was accomplished by the condiagonalization. The method also relied on the use of $\kappa^{\mathrm{cc}}$ since the minimal condition for disentangling background and resonance scatterings was provided by $\mathfrak{R}\left(\mathrm{K}^{\mathrm{LL}}\right)=0$.

Application of the Theory to the Study of Overlapping Resonances in Rare Gas Spectra. The resonance structures in the Lu-Fano plot shown in Fig. 3 for $J=1$ states of argon at the lowest ionization thresholds ${ }^{2} \mathrm{P}_{3: 2}^{\mathrm{P}}$ and ${ }^{2} \mathrm{P}_{1: 2}^{0}$, were drawn using the NIST atomic spectra database. ${ }^{41}$ Theoretical Lu-Fano curves were obtained using the $t_{\text {na }}$ values reported by Lee and $\mathrm{Lu}^{2}$ with $\mu_{\alpha}$ values modified to $[0.20,0.07,0.48,0.15,0.12]$ for better fitting to the experimental data. The resonance structures were first compared using the eigenquantum-defect sums in

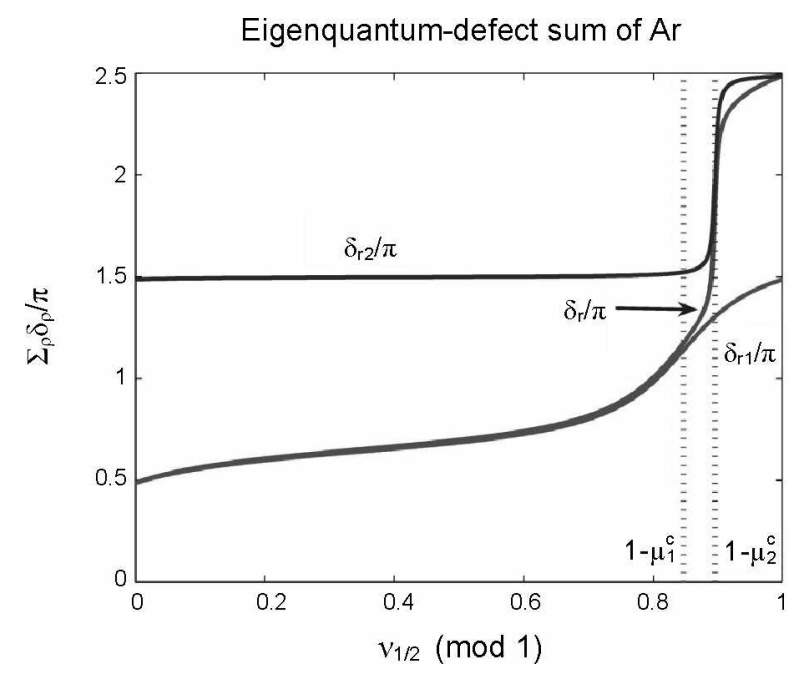

Figure 5. Decomposition of the sum of the eigenquantum-defects extracted from the Lu-Fano plot for the $J=1$ states of argon at the lowest ionization thresholds ${ }^{2} \mathrm{P}_{3: 2}^{0}$ and ${ }^{2} \mathrm{P}_{1: 2}^{\circ}$.

the original and con-diagonalized representations shown in Figs. 4 and 5 , respectively. Although the difference in the eigenquantum-defect sums is difficult to see in the graphs in both figures. a completely different situation would result if $\delta_{\mathrm{t} 1}+\delta_{\mathrm{t} z}-\delta_{\mathrm{i}}$ were compared. The sumunation relation. $\delta_{\mathrm{t}}=\delta_{i 1}+$ $\delta_{\text {t. }}$ does not hold as shown in Fig. 4 while it holds in Fig. 5. Note that in order to draw Fig. 5. an isolated resonance case was assumed so that only the first term of the right-hand side of Eq. (12) is included.

Two contributions to the sum of the eigenquantum defects can be identified in Figs 4 and 5 : the increase from $\sim 0.5$ to $\sim 1.5$ by one in the first half is due to $\delta_{\mathrm{rl}}$ and the increase from $\sim 1.5$ to -2.5 by one in the second half is due to $\delta_{\mathrm{t}}$. The overall increase of 2 in the quantum defect means that there are two resonances contributing in one unit interval of $v_{1: s}$, the $d$ and $s$ Rydberg series. respectively:

The resonance structures can be made conspicuous using time delay's. ${ }^{48.99}$ The time-delay's were calculated from $\tau(E)=$ $2 \hbar d \delta(E) / d E$ where $E$ is related to $v_{12}$ by $I_{1: 2}-\operatorname{Ryd} / w_{1: 2}$ in the present system. Note that time delays would be due to resonance scattering alone if the energy dependence of background scattering can be neglected. As before. the time delays, $\tau_{j_{1},}, \tau_{\mathrm{j}_{2}}$ and $\tau_{\mathrm{T}}$ due to resonance scatterings are shown in Fig. 6 for both the original and con-diagonalized representations. Although both representations yielded almost identical total time delays, the time delays due to the $s$ series were different. which was expected because the sunmuation relation $\tau=\tau_{1 j}+\tau_{j_{2}}$ holds only for the con-diagonalized representation while not for the original one.

The resonance structures in the krypton and senon spectra were also analyzed using the $U_{\text {ia }}$ values reported by Geiger ${ }^{2,1}$ and Dill ${ }^{-\otimes}$ with $H_{\alpha}$ values modified to $[0.235,0.097,0.47,0.12$, $0.07]$ and $[0.36 .0 .12 .0 .56,0.040 .-0.007]$, respectively, for the best fit to the experimental data. The results for $\mathrm{Kr}$ and $\mathrm{Xe}$ are showin. respectively. in Tables 4 and 5 and Figs. 7 and 8. The resonance structures in both spectra are similar. showing two resonance structures due to the $s$ and $d$ Rydberg series. The differences in the positions of the resonance structures 
Time delays of Ar in con-diagonalized representation
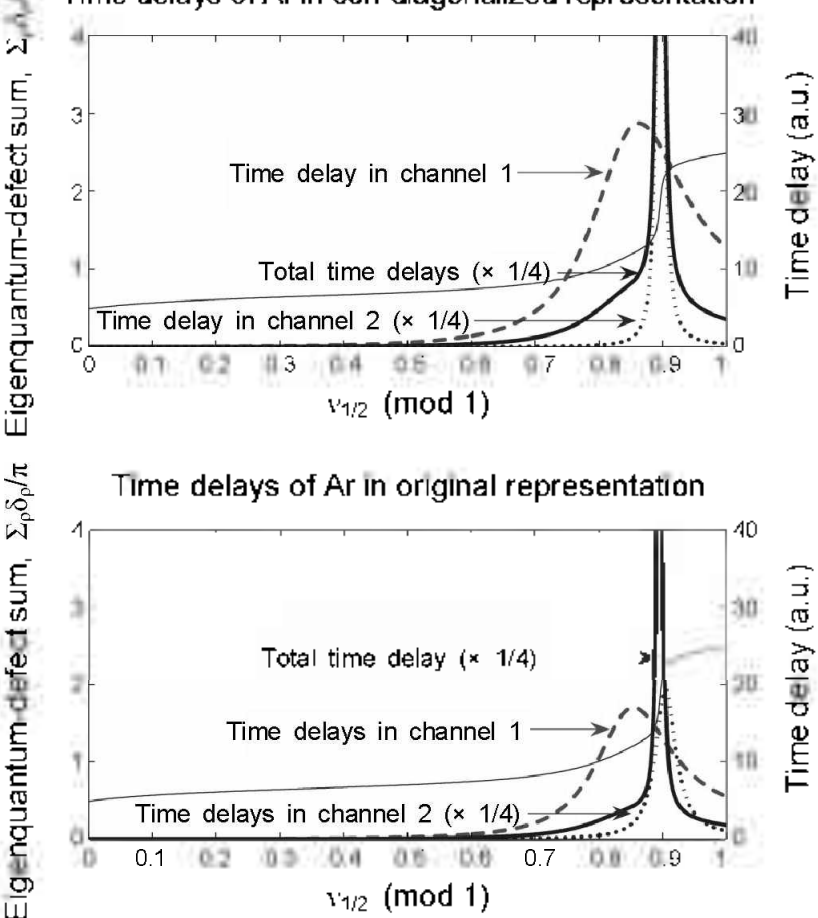

Figure 6. IDeemposition of the tume delays in the orignal and con-diagonalized representations extracted from the l.u-l ano plot for the $I$ - I states of argen at the lonest ionization thresholds "P?" and $-\mathrm{P}^{2}$ ")

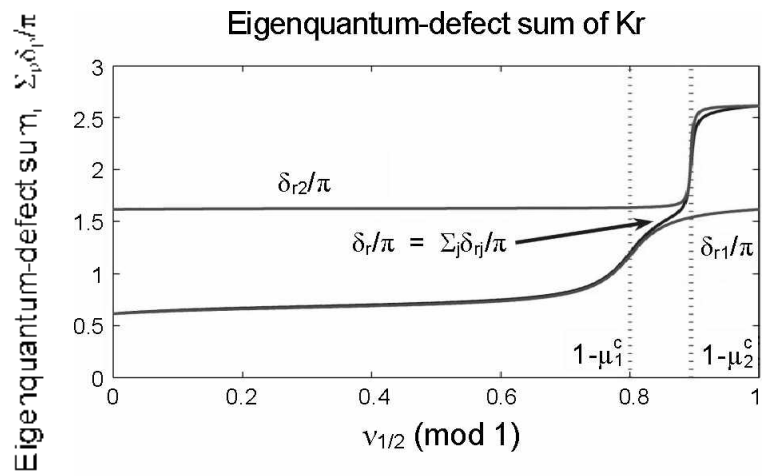

Lu-Fano plot of $\mathrm{Kr}$

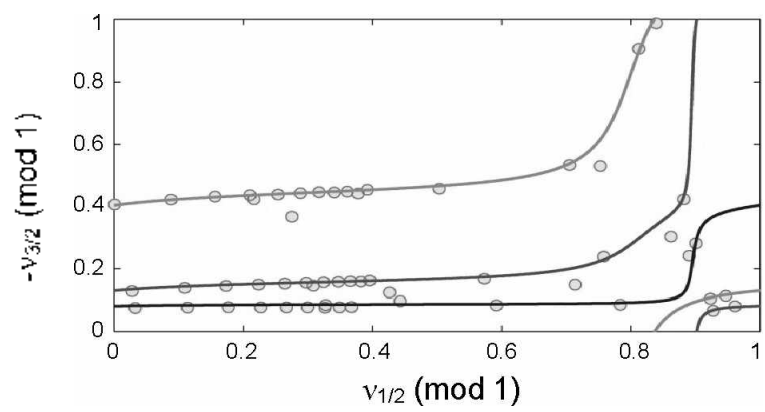

Figure 7. Jeenmposition of the sum of the eigencuantum-defects and the Iol-Fano plot for the $J=1$ states of krypton at the lowest ionization thresholds " ${ }^{2} 3$ and ${ }^{2} P^{2} 12$.

can be measured by the difference in $\mu$ and $\mu$. Their values increase from 0.04 in argon, to 0.09 and 0.25 in krypton and

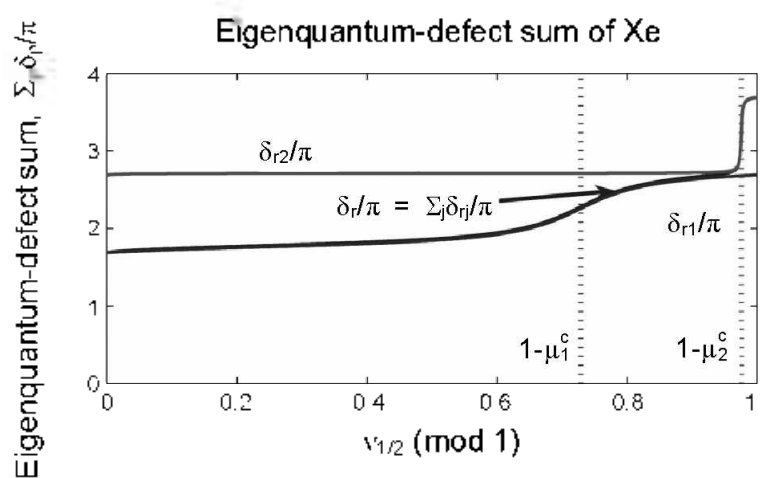

Lu-Fano plot of $\mathrm{Xe}$

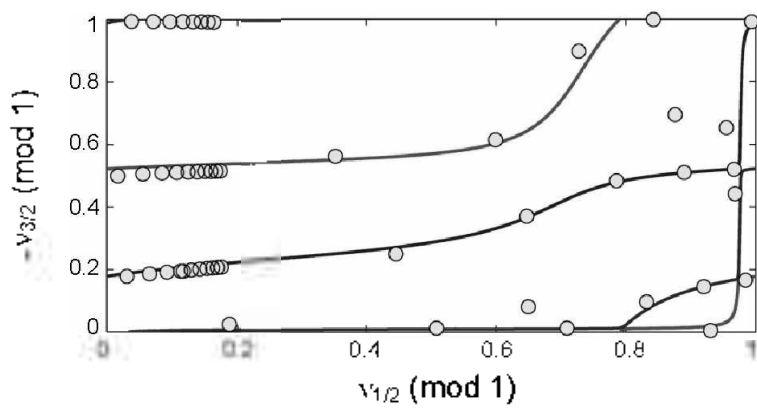

Figure 8. I tecomposition of the sum of the cigencuentun-deliets and $L$ d-tano plot tot the $J-I$ states of xenon at the lotsest ionization thresholds "P?" and "lp":

xenon, respectively: The contributions of the overlapping resonances can be obtained by calculating the extent of the defect from unitv of $V^{\mathrm{rl}} V$. or more conveniently from its trace $\operatorname{tr}\left(V^{\mathrm{T}} \mathrm{l}\right)$. Calculaicd values for 3 spectra are included in Tables 3.4 and 5 , respectively. Note that values arc close to 2 . meaning that resonances in these rare gas spectra are almost isolated. However, the overlapping cannol be neglected completels. Wintgen and Friedrich related almost non-overlapping to the vanishing widths. here. of the $s$ series." Interestingl: the deviation from unity [or from 2 for $\operatorname{tr}\left(l^{\text {pr }} V\right)$ ] was largest in kryplon instead of argon, where two resonance structures were located in close proximity. This anomaly is in line with the behavior in the variation of the values of the coupling strengths $\left(\tilde{E}_{1}^{2}, Z_{2}^{2}\right)$ in the $d$ and $s$ series which are given by $(0.18,0.0057)$. (0. 14, 0.009) 1 ) and $(0.25,0.0051)$ from Tables 3-5 for argon. krypton and xenon, respectively. The physical nature of these phenomena will not be examined further because the purpose of this paper was to set up an analysis tool of resonance structures.

The resonance structures in the autoionization spectra of $\mathrm{Ar}, \mathrm{Kr}$ and $\mathrm{Xc}$ in Figs. 9, 10 and 11, respectively were analy zed using this method. The anal sis revealed a one-to-one correspondence of the resonance structures in the autoionization spectra with the ones manifested in time delars. The overall shapes were quite similar in the autoionization spectra and time delays. However. their detailed structures differed. cren qualitatively: The difference was more conspicuous in the $d$ Rrdberg series. The increase in the time delays with $v_{1}$ comes from the factor $v_{1}^{3}=$ and reflects the increase in orbiting period. 
Autoionization profile of $\mathrm{Ar}$

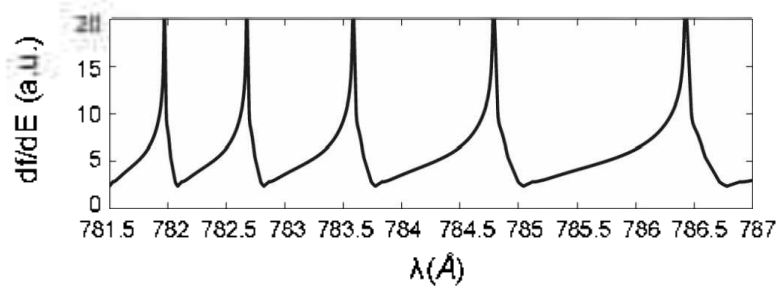

Time delays in $s$ and $d$ series of $\mathrm{Ar}$
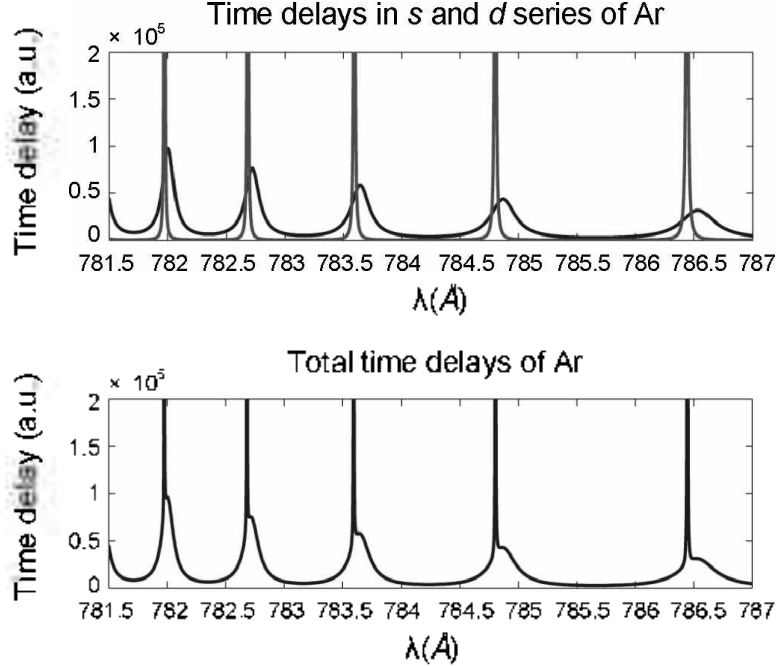

Figure 9. Oscillator strength densities and time delays of the autoionization lines in the argon spectra between the lowest ionization thresholds " $P$ ?
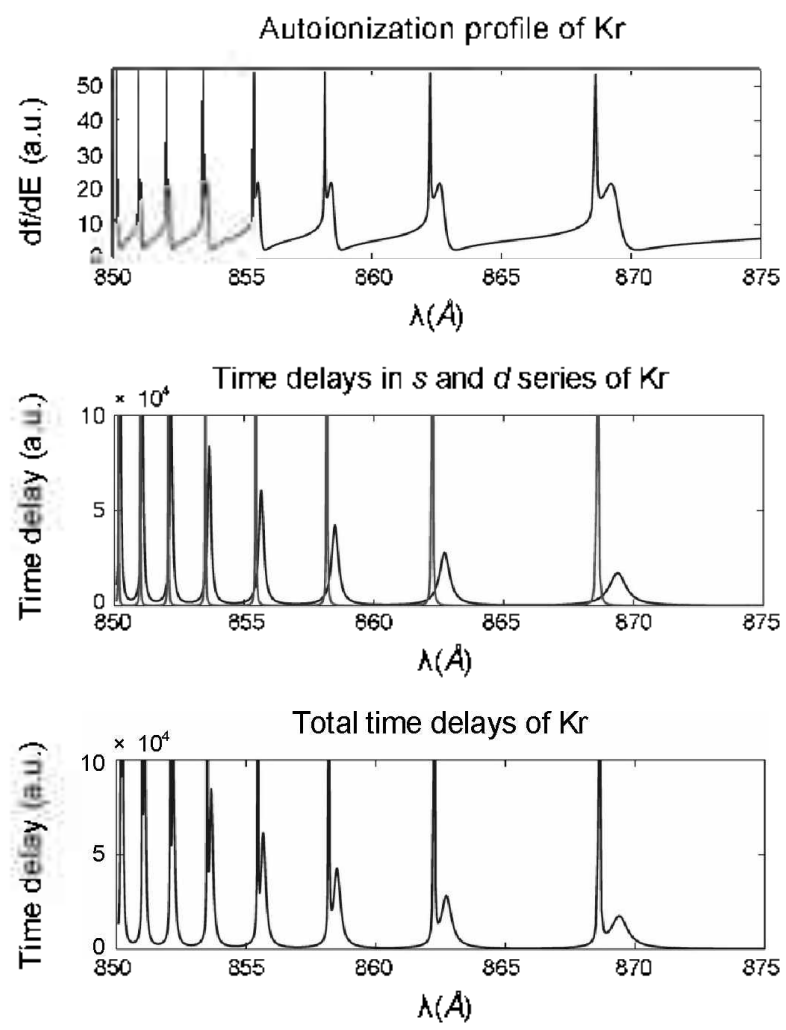

Figure 10. Oscillator strength densities and time delas sof the autoionization lines in the kry pton spectra between the lowest ionization thresholds ${ }^{-P}+2$ and ${ }^{2} P^{\prime \prime}=$
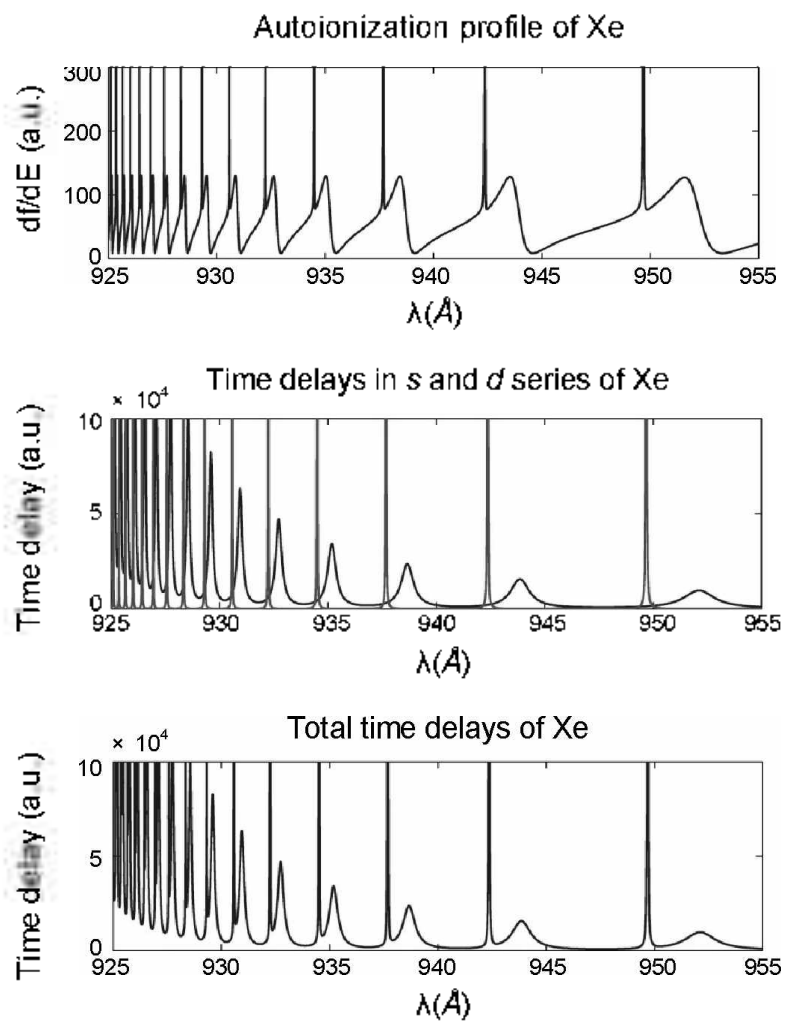

Figure 11. Oscillator strengh densities and time delays of the attoionization lines in the xenon spectra beween the lowest ionization thresholds ${ }^{2} P_{3}^{0}=$ and ${ }^{2} P_{1: 2}^{*}$

\section{Summary and Discussion}

Previously, a methodology was developed for identifying the background and resonance channels and for finding phase renormalizations in those channels, but not in the open and closed channels which are incompatible with the background and resonance channels. Application of this theory to the $\mathrm{Ne}$ and Kr Lu-Fano plots showed quite different but more meaningful coupling strengths between the open and closed charnels in the representation, where the background scattering was disentangled from resonance scattering.

This theory was extended to the cases involving the degenerate closed channels, which is interesting in that it is the simplest system where overlapping resonances are present and proper handlings are thus required. The case involving degenerate closed channels is special in that $\tan \beta$ is a constant matrix and commutes with any matrix. Identification of the resonance eigenchannels involves diagonalization of the nonI Iermitian (or, more properly, normal) symmetric complex matrix $\kappa^{\text {te }}$. As non-normal symmetrical complex matrix can only be con-diayonalized unitarily, $\kappa^{\mathrm{cL}}$ car be factorized to $V^{\mathrm{C}} \mathrm{K}^{\mathrm{CL}} V^{\mathrm{I}}$. It was found that the isolated resonances correspond to $\kappa^{\mathrm{ut}}$ normal. Therefore, deviation from normality, which was measured by the defect of $V^{1} V$ from unity, was used to handle the overlapping resonances in the MQDT formulation systematically. The theory on con-diagonalization and con-similarity and Takagi's factorization is essential for handling the overlapping resonances, 
In order to compare the present method with the established method of making $K^{-\infty}$ and $K^{-c c}$ null. resonance structures were derived when $K^{-09}$ and $K^{-c c}$ are null matrices. Resonance structures thus obtained were complicated so that individual resonance contribution was not clearly discernible. indicating that they were not disentangled yet. Thus naking $K^{\circ}$ and $K^{\circ c}$ null was found not to be sufficient to guarantee the separation of background and resonance scatterings and identification of resonance structures in the presence of resonance overlapping. This strange result derived essentially from the impossibility of disentangling the background and resonance scatterings when overlapping resonances are present. The difficulty was avoided in this work at first by separating isolated and overlapped terns using condiagonalization.

The theory was applied to the rare gas spectra of $\mathrm{Ar} . \mathrm{Kr}$ and $\mathrm{Xe}$ and the overlapping resonances present in their spectra were analyzed. The resonance structures in the $s$ and $d$ series in $\mathrm{Ar}, \mathrm{Kr}$ and $\mathrm{Xe}$ spectra were separated and the relevant dynanuc parameters were extracted. The separations in the resonance positions, which were measured by the extent of the phase renormalizations in the closed channels, increased from $\mathrm{Ar}$ to $\mathrm{Xe}$. However the coupling strengths showed a different behavior. Their magnitudes were the maxinum and minimum in $\mathrm{Kr}$ in the $s$ and $d$ series. respectively. Overlapping between resonant structures was found to be virtually absent in all spectra. It was largest in the $\mathrm{Kr}$ spectra. showing similar tendency to the coupling strengths between the resonance and background scatterings. Although the line profiles were not analyzed. Figs. 9-11 suggest that they take rather symmetric shapes in the disentangled $s$ and $d$ series, which is in contrast to the apparent asy'mmetric appearance in the autoionization lines profiles. particularly in the $d$ series.

Acknowledgments. This study was supported by the Ajou university research fellowship of 2008 under contract No. 20083970COR0101S000100. One of the authors (C.-W. Lee) is greatly thankful to Professor Kiyoshi Ueda for his kind explanation about his work and telling me some untold stories in the alternative parameterization of MQDT.

\section{Appendix A: The Derivation of Eq. (14)}

Since the phase $\delta_{\mathrm{r}}^{\prime}$ is defined as the one of $\operatorname{det}\left(\tan \beta^{\prime}+\kappa^{\prime \prime c}\right.$ ), its tangent is given as a ratio of the detenninant's real and inaginary parts:

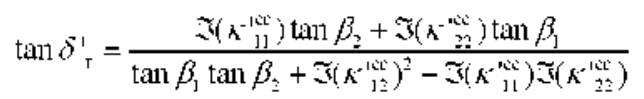

where $\beta_{1}$ and $\beta_{2}$ denote $\beta+\pi t_{1}^{*}$ and $\beta+\pi \mu_{-}^{\circ}$, respectively. Using the trigonometric relation $\tan \left(\beta+\pi_{t} t_{i}^{c}\right)=\left(\tan \beta+\tan \pi_{i} t_{j}^{c}\right) /(1-\tan \beta$ tan $\pi u_{i}^{i}$, both numerator and denominator can be expressed as quadratic functions of $\tan \beta$ so that $\tan \delta_{i}^{\prime}$ can be written as

$$
\tan \delta^{\prime},=\frac{n_{2} \tan ^{2} \beta+n_{1} \tan \beta+n_{0}}{d_{2} \tan ^{2} \beta+d_{1} \tan \beta+d_{0}}
$$

where parameters in the numerator denote

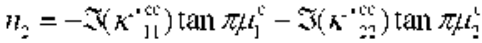

$$
\begin{aligned}
& \left.n_{1}=\operatorname{tr} \Im\left(K^{*}\right)(1) \tan \pi \mu_{1}^{c} \tan \pi \mu_{2}^{c}\right)
\end{aligned}
$$

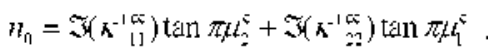

and ones in the denominator denote

$$
\begin{aligned}
& d_{2}=1-\operatorname{det} \pi\left(k^{\prime<c}\right) \tan \pi \mu_{1}^{c} \tan \pi \mu_{2}^{c} \\
& d_{1}=\left(\tan \pi \mu_{1}^{c}+\tan \pi \mu_{2}^{i}\right)\left[1+\operatorname{det} \Im\left(\kappa^{\prime} c c\right)\right] \\
& d_{0}=\tan \pi \mu_{1}^{i} \tan \pi \mu_{2}^{c}-\operatorname{det} \Im\left(\kappa^{\prime<i}\right)
\end{aligned}
$$

Eq. (Al) can be easily transformed into Eq. (14) where $r_{1}, r_{2}, s_{1}$ and $s_{1}$ are obtained as follows:

$$
\begin{aligned}
& r_{1.2}=\frac{d_{1} \pm \sqrt{d_{1}^{2}-4 d_{0} d_{2}}}{2 d_{2}}, \\
& \left(\begin{array}{l}
s_{1} \\
s_{2}
\end{array}\right)=\frac{1}{d_{2}\left(r_{1}-r_{2}\right)}\left(\begin{array}{c}
r_{1}\left(n_{1}-n_{2} d_{1} / d_{2}\right)-n_{0}+n_{2} d_{0} / d_{2} \\
\left.-i_{2}\left(n_{1}-n_{2} d_{1} / d_{2}\right)+n_{0}-n_{2} d_{0} / d_{2}\right)
\end{array}\right) .
\end{aligned}
$$

\section{References}

1. Seaton, M. T. Rep. Prog. Phus 1983, $\$ 6,167$.

2. Fano, U.: Rau, A. R. P. Atomic Collisions and Spectra; Academic: Orlando, U. S. A., 1986.

3. Jungen, C. Molecular Applications of Quntum Defect Theory; Institute of Physics: Bristol, UK. 1996.

4. Eissner, W.: Nussbaumer, H.; Saraph, H. E.; Seaton, M. J. $J$. Phs B 1969, 2, 341

5. Cooke, W. E.; Cromer, C. L. Plys. Rew A 1985, 32, 2725

6. Giusti-Suzor, A:; Fano, U. J. Phvs. B 1984, 17, 215.

7. Giusti-Suzor, A.; Lefebvre-Brion, H. Phys Rev it 1984, 30 , 3057

8. Ueda, K. Pins. Rev. A 1987, 35, 2484.

9. Lecomte, J. M. J. Phvs. B 1987, 20, 3645.

10. Wintgen, D.; Friedrich, H. Phys. Rev. A 1987, 35, 1628.

11. Fano, U. Phus Rev 1961, 124, 1866.

12. Lee, C.-W. Bull. Korean Chent Soc. 2002, 23, 971

13. Lee, C.-W.; Kim, T.-H. Bull. Korean Chem. Sac, 2002, $23,1560$.

14. Lee, C. -W. Phys Rev A 2002, 66, 052704.

15. Lee, C.-W. Bull. Korean Chem. Soc. 2009, 30,891.

16. Wigner. E. P. Am Math $1951,53,36$.

17. Feshbach, H. Ant Phys A.Y, 1967, t3, 410 .

18. Mies, F. H. Phtys. Rev $1968,175,164$.

19. McVoy, K. W. Am. Phys. (N. Y. 1969, 54, 552

20. Simonius, M. Aucl Phys A $1974,218,53$

21. Lyuboshitz, V. L. Phys. Lett. B 1977, 72, 41 .

22. Connerade, J. P.; Lane, A. M. Rep. Prog. Phys. 1988, $51,1439$.

23. Magunov, A. I.: Rotter, I.: Strakhova, S. I. Phis. Rev B 2003, 68 , 245305

24. Tabanli, M. M.; Peacher, I. L.: Madison, D. H. J. Phys. B 2003, 36,217

25. Lu, K. T.; Fano, U. Phys. Rev A 1970, 2,81 .

26. Lu, K. T. Phus Rev A $1971,4,579$.

27. Lee, C. M; Lu, K. T. Phys. Rev .4 1973, 8, 1241.

28. Dill, D. Phys. Rev A 1973, 7, 1976.

29. Geiger, T. Z. Phys .4 1976, 276, 219

30. Geiger, T. $Z$. Phus A $1977,282,129$

31. Iohnson, W. R.: Cheng, K. T.; Huang, K. N.; Le Doumeuf, M. Phus. Rev. A 1980, 22, 989. 
32. Aymar, M: Robaus, O: Thomas, C. J. Phss. B 1981, 14, 4255.

33. Klar, D.: Aslam, M.; Baig, M. A.: Ueda, K.; Ruft, M. W.: Hotop, H. J. Phys. B 2001, 34, 1549 .

34. Baig, M. A.; Hanif, M.; Aslam, M.; Bhatti, S. A. J. Phys. B 2006, 39,4221

35. Liang, L.: Jiang, W. X.: Zhou, C.: Zhang, L. Opt. Comm. 2008, 281,2107

36. Wright, T. D : Morgan, T. T.; Li, L. P: Gu, Q. L.; Knee, J. L.; Petrov, I. D.; Sukhorukov, V. L.; Hotop, H. Phus. Rev. A 2008, 77,062512 .

37. Ueda, K. J. Opt. Soc. Am. B 1987, 4, 424.

38. Mullins, O.C.; Zhu, Y.; Xu, E. Y.; Gallagher, T. F. Phns. Rev. A $1985,32,2234$

39. Ginsti-Suzor, A.; Fano, U. J. Phvs. B 1984, 17, 4277.
40. Dubau, I.: Seaton, M. J. J. Phss. B 1984, 17, 381.

41. Ralchenko, Y.; Kramida, A. E.; Reader, J:: NIST ASD Team, NIST Afomic Spectra Database (version 3.1.5): National Institute of Standards and Technology: Gaithersburg, U. S. A., 2008.

42. Macklin, P. A. Ant. J. Phys. 1984, 52,513

43. Horn, R. A.: Iohnson, C. R. Matrix Analysis; Cambridge: Cambridge, U. K. 1985.

44. Muir, T. A Treatise on the Theory of Detemtinants, Dover: New York, U. S. A., 1960.

45. Fano, U. Rev Mod. Phys 1957, 29, 74.

46. Lee, C.-W. Phus Essans 2000, 13, 206.

47. Fano, U.; Cooper, T. W. Phvs. Rev A 1965, 137, 1364.

48. Smith, F. T. Phys. Rew 1960, 118, 349.

49. Lippmann, B. A. Phys. Rev. 1966, 151, 1023. 